\title{
Material stability analysis of particle methods
}

\author{
S.P. Xiao ${ }^{a}$ and T. Belytschko ${ }^{\text {b,* }}$ \\ ${ }^{a}$ Department of Mechanical and Industrial Engineering and Center for Computer-Aided Design, \\ The University of Iowa, 3131 Seamans Center, Iowa city, IA 52242, USA \\ E-mail: sxiao@engineering.uiowa.edu \\ ${ }^{b}$ Department of Mechanical Engineering, Northwestern University, $2145 \mathrm{~N}$ Sheridan Rd, Evanston, \\ IL 60208, USA
}

Received 8 April 2003; accepted 20 December 2003

Communicated by Z. Wu and B.Y.C. Hon

\begin{abstract}
Material instabilities are precursors to phenomena such as shear bands and fracture. Therefore, numerical methods that are intended for failure simulation need to reproduce the onset of material instabilities with reasonable fidelity. Here the effectiveness of particle discretizations in reproducing of the onset of material instabilities is analyzed in two dimensions. For this purpose, a simplified hyperelastic law and a Blatz-Ko material are used. It is shown that the Eulerian kernels used in smooth particle hydrodynamics severely distort the domain of material stability, so that material instabilities can occur in stress states that should be stable. In particular, for the uniaxial case, material instabilities occur at much lower stresses, which is often called the tensile instability. On the other hand, for Lagrangian kernels, the domain of material stability is reproduced very well. We also show that particle methods without stress points exhibit instabilities due to rank deficiency of the discrete equations.
\end{abstract}

Keywords: particle methods, stability, Lagrangian kernels

AMS subject classification: 74 S30

\section{Introduction}

Particle methods are a class of meshfree methods. The original particle methods was based on kernel approximations [20]. In some cases, as shown by Belytschko et al. [4], kernel methods are closely related to the mesh-free methods that are based on field approximations. However, the kernel approximations used in particle methods are somewhat inaccurate because they cannot exactly reproduce linear functions. With corrected derivative methods, as developed by Randles and Libersky [23] and Krongauz and Belytschko [17], the kernel approximations are corrected so that they can reproduce derivatives of linear functions exactly. Alternatively, Liu et al. [18] has developed correction functions that enable particle methods to reproduce linear functions exactly; these

\footnotetext{
* Corresponding author.
} 
corrected particle methods are identical to the moving least square (MLS) approximation with a linear basis as used in the element-free Galerkin (EFG) methods [6].

Material instabilities occur in nonassociative plasticity and softening materials where stress decreases with increasing strain. The literature on material instability goes back at least as far as Hadamard [13] who examined the question of what happens when the tangent modulus is negative. He identified the conditions for a vanishing propagation speed of an acceleration wave as a material instability. In 1962, Hill [14] made a general analysis of acceleration waves and stability. Other relevant works are [25,26].

Material instability is usually associated with a localized growth of the deformation, called localization. It corresponds to the phenomena that are observed in nature where narrow bands of intense deformation occur in failure processes. These are often called shear bands, since the deformation mode in these bands is usually shear $[15,19]$.

Material instability in equilibrium problems is associated with loss of ellipticity of the incremental equations of equilibrium. As shown in [21], loss of ellipticity will always occur when the tangent modulus loses positive definiteness, but can occur even for a positive definite tangent modulus. In the dynamic equations, tangent moduli that violate ellipticity conditions lead to loss of hyperbolicity, so that three real positive wavespeeds no longer occur in every direction. This is also indicative of a material instability.

In addition to material instability, particle methods have their own numerical instability [3,7]. For nodal quadrature as proposed by Beissel and Belytschko [2], where integrals are evaluated by summing the function at the nodes, there is an additional instability due to rank deficiency of the discretization of the divergence of the stress. This is also found in underintegrated finite elements: in quadrilateral elements, these spurious modes are called hourglass modes. Rank deficiency leads to singular discrete equilibrium equations and occurs regardless of the state of stress. Stabilization schemes can be developed for rank deficiency, as in finite element, see [12]. One approach to stabilization is to use additional quadrature points called stress points or slave points as proposed by Dyka et al. $[10,11]$. With a proper arrangement of particles, stress points can eliminate the instability due to the rank deficiency. However, the material instability cannot, and should not be eliminated.

Kernels used in particle methods can be classified as Lagrangian and Eulerian. Lagrangian kernels are functions of the material (or Lagrangian) coordinates and Eulerian kernels are functions of the spatial (or Eulerian) coordinates. In 1D we have found that Eulerian kernels do not reproduce the material instability accurately [7]. In fact, the well known tensile instability [27] is a manifestation of error in the reproduction of material instability by Eulerian kernels. The Lagrangian kernel, however, reproduces the onset of material instability exactly in 1D. Randles et al. [24] investigated the stability of dual particle dynamics and smooth particle dynamics. They found that linear completeness led to stability; their conclusions differ from ours as discussed in section 9.

In this paper we examine how closely Lagrangian and Eulerian kernels reproduce the onset of material instabilities in two dimensions. For this purpose, it is necessary to choose a specific material law. Therefore, we have used a hyperelastic material, which is similar to the Mooney-Rivlin material, and a Blatz-Ko material. Linearized stability 
theory is used. An infinite body is considered in a state of uniform initial stress and deformation and the response to small perturbations is examined.

The outline of this paper is as follows: In section 2, we present our notation and the basic equations; we also indicate why we can study an isotropic material by considering a diagonal deformation matrix. In section 3 a hyperelastic material similar to a Mooney-Rivlin material is given. Then we obtain the domain of material stability for this hyperelastic material based on a linearized stability analysis; this is the domain of material stability of the governing partial differential equations (PDEs). In section 5, stability analyses of particle methods with different integration methods and different kernel functions are made for the hyperelastic material and the stable domains are compared to that of the PDEs. The stable domain for the PDEs and for particle discretizations for the Blatz-Ko material are developed in section 8. Section 9 gives conclusions.

\section{Notation, basic principles and governing equations}

To simplify the analysis, we show that for an isotropic material, it suffices to consider a diagonal deformation gradient $\mathbf{F}$ for the initial state of deformation.

As shown in figure 1 , we denote the material coordinates by $\mathbf{X}$, the reference configuration by $\Omega_{0}$, the spatial coordinates by $\mathbf{x}$, and the current configuration by $\Omega$. The base vectors of a Cartesian system are denoted by $e_{i}$, so $\mathbf{X}=X_{i} e_{i}$. A motion is given by the mapping $\mathbf{x}=\mathbf{x}(\mathbf{X}, t)$. The map must be one to one except on sets of measure zero (e.g., fracture surfaces in 3D), where points can have multivalued motions to model phenomena such as cracking.

The governing equation in the reference configuration is

$$
\rho_{0} \ddot{\mathbf{u}}=\nabla_{0} \cdot \mathbf{P}
$$

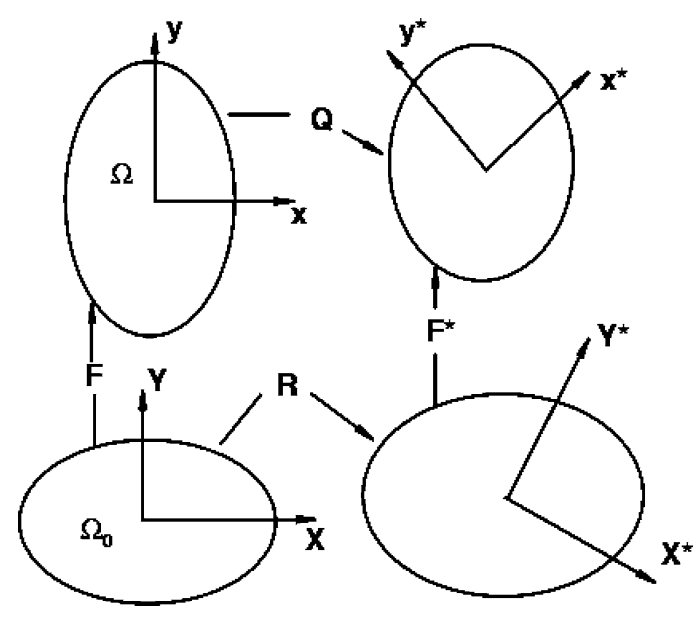

Figure 1. Deformed configurations. 
where $\rho_{0}$ is the initial density, $\mathbf{P}$ is the nominal stress tensor, $\mathbf{u}$ is the displacement, $\nabla_{0}$ is the gradient with respect to the material coordinate $\mathbf{X}$ and the superposed dots denote material time derivatives.

We will consider an initial, homogeneous state of deformation so the motion can be written as $\mathbf{x}=\mathbf{F X}$, where the deformation gradient $\mathbf{F}$ is constant (we omit the constant of translation). The right Cauchy-Green deformation tensor is $\mathbf{C}=\mathbf{F}^{\mathrm{T}} \mathbf{F}=\mathbf{U} \mathbf{U}$. By using a spectral decomposition, since $\mathbf{C}$ is a positive definite symmetric matrix, we can find a rotation tensor $\mathbf{R}$ which can diagonalize $\mathbf{C}$ so that $\mathbf{C}^{*}=\mathbf{R}^{\mathrm{T}} \mathbf{C R}=\mathbf{U}^{*} \mathbf{U}^{*}$ is a diagonal matrix where $\mathbf{X}^{*}=\mathbf{R}^{\mathrm{T}} \mathbf{X}$. If we let $e_{i}^{*}=\mathbf{R} e_{i}$, then in terms of the basis $e_{i}^{*}$

$$
\mathbf{C}^{*}=\left[\begin{array}{ccc}
\lambda_{1}^{2} & 0 & 0 \\
0 & \lambda_{2}^{2} & 0 \\
0 & 0 & \lambda_{3}^{2}
\end{array}\right], \quad \mathbf{U}^{*}=\left[\begin{array}{ccc}
\lambda_{1} & 0 & 0 \\
0 & \lambda_{2} & 0 \\
0 & 0 & \lambda_{3}
\end{array}\right]
$$

and the corresponding deformation gradient is

$$
\mathbf{F}^{\prime}=\frac{\partial \mathbf{x}}{\partial \mathbf{X}^{*}}=\frac{\partial \mathbf{x}}{\partial \mathbf{X}} \frac{\partial \mathbf{X}}{\partial \mathbf{X}^{*}}=\mathbf{F R} .
$$

Using the polar decomposition $\mathbf{F}^{\prime}=\mathbf{Q} \mathbf{U}^{*}$, where $\mathbf{Q}$ is another rotation matrix which relates $\mathbf{x}$ to $\mathbf{x}^{*}$ by $\mathbf{x}=\mathbf{Q \mathbf { x } ^ { * }}$, we arrive at the following

$$
\mathbf{F}^{*}=\frac{\partial \mathbf{x}^{*}}{\partial \mathbf{X}^{*}}=\frac{\partial \mathbf{x}^{*}}{\partial \mathbf{x}} \frac{\partial \mathbf{x}}{\partial \mathbf{X}} \frac{\partial \mathbf{X}}{\partial \mathbf{X}^{*}}=\mathbf{Q}^{\mathrm{T}} \mathbf{F R}=\mathbf{U}^{*}=\left[\begin{array}{ccc}
\lambda_{1} & 0 & 0 \\
0 & \lambda_{2} & 0 \\
0 & 0 & \lambda_{3}
\end{array}\right] .
$$

Thus, we can characterize the initial state of deformation by three stretches $\lambda_{i}$. We will use this deformation gradient for the initial state of the body in the stability analysis, although our analyses will be limited to two dimensions, so we will use only upper left $2 \times 2$ submatrix of (4).

\section{Material model}

An isotropic hyperelastic material [5] model is first considered here. Its potential is given by

$$
\psi=\frac{1}{2} c_{1} I_{1}+\frac{1}{2} c_{2} I_{2}-\left[\frac{3}{2} c_{1} I_{3}^{1 / 3}+\frac{3}{2} c_{2} I_{3}^{2 / 3}-\frac{\lambda}{4}\left(\ln I_{3}\right)^{2}\right],
$$

where $I_{1}, I_{2}$ and $I_{3}$ are the principal invariants of the right Cauchy-Green deformation tensor $\mathbf{C}$. This model is similar to the Mooney-Rivlin material except that the MooneyRivlin material is incompressible, i.e. $I_{3}=1$. We use this as a model material because it develops material instability more readily than a Mooney-Rivlin material.

The first derivatives of the principal invariants are:

$$
\frac{\partial I_{1}}{\partial \mathbf{C}}=\mathbf{I}, \quad \frac{\partial I_{2}}{\partial \mathbf{C}}=I_{1} \mathbf{I}-\mathbf{C}^{\mathrm{T}}, \quad \frac{\partial I_{3}}{\partial \mathbf{C}}=I_{3} \mathbf{C}^{-\mathrm{T}} .
$$


The second Piola-Kirchhoff stress tensor $\mathbf{S}$ is given by

$$
\mathbf{S}=2 \frac{\partial \psi}{\partial \mathbf{C}}=\left(c_{1}+c_{2} I_{1}\right) \mathbf{I}-c_{2} \mathbf{C}-\left(c_{1} I_{3}^{1 / 3}+2 c_{2} I_{3}^{2 / 3}-\lambda \ln I_{3}\right) \mathbf{C}^{-1} .
$$

The tangent modulus tensor $\mathbf{C}^{\mathrm{SE}}$, which is also called the second elasticity tensor, relates the rate of the second Piola-Kirchhoff stress to the Green strain rate by $\dot{\mathbf{S}}=$ $\mathbf{C}^{\mathrm{SE}}: \dot{\mathbf{E}}$. It is given by

$$
\mathbf{C}^{\mathrm{SE}}=2 \frac{\partial \mathbf{S}(\mathbf{C})}{\partial \mathbf{C}}=2 c_{2} \mathbf{I} \otimes \mathbf{I}-2 c_{2} \mathbf{I}-2 s_{0} \frac{\partial \mathbf{C}^{-1}}{\partial \mathbf{C}}-2 s_{1} \mathbf{C}^{-1} \otimes \mathbf{C}^{-1},
$$

where

$$
s_{0}=c_{1} I_{3}^{1 / 3}+2 c_{2} I_{3}^{2 / 3}-\lambda \ln I_{3} \quad \text { and } \quad s_{1}=\left(\frac{1}{3} c_{1} I_{3}^{-2 / 3}+\frac{4}{3} c_{2} I_{3}^{-1 / 3}-\frac{\lambda}{I_{3}}\right) I_{3} .
$$

Writing (8) in indicial form, the tangent modulus tensor is given by

$$
C_{i j k l}^{\mathrm{SE}}=2 c_{2} \delta_{i j} \delta_{k l}-c_{2}\left(\delta_{i k} \delta_{j l}+\delta_{i l} \delta_{j k}\right)+s_{0}\left(C_{i k}^{-1} C_{j l}^{-1}+C_{i l}^{-1} C_{j k}^{-1}\right)-2 s_{1} C_{i j}^{-1} C_{k l}^{-1} .
$$

The spatial form of the second elasticity tensor is the fourth elasticity tensor $\mathbf{C}^{\tau}$ and it relates the convected rate of Kirchhoff stress to the rate-of-deformation, i.e. $\boldsymbol{\tau}^{\nabla c}=$ $\mathbf{C}^{\tau}: \mathbf{D}$. It is given by

$$
C_{i j k l}^{\tau}=F_{i m} F_{j n} F_{k p} F_{l q} C_{m n p q}^{\mathrm{SE}} .
$$
ation is

The relation between the Truesdell rate of the Cauchy stress and rate-of-deform-

$$
\boldsymbol{\sigma}^{\nabla \mathrm{T}}=J^{-1} \mathbf{C}^{\tau}: \mathbf{D}=\mathbf{C}^{\sigma \mathrm{T}}: \mathbf{D},
$$

where the Truesdell rate of Cauchy stress is given by

$$
\boldsymbol{\sigma}^{\nabla \mathrm{T}}=\dot{\boldsymbol{\sigma}}+\operatorname{div}(\mathbf{v}) \boldsymbol{\sigma}-\mathbf{L} \cdot \boldsymbol{\sigma}-\boldsymbol{\sigma} \cdot \mathbf{L}^{\mathrm{T}}
$$

and

$$
\boldsymbol{\sigma}=\frac{1}{J} \mathbf{F} \cdot \mathbf{S} \cdot \mathbf{F}^{\mathrm{T}}, \quad \mathbf{L}=(\nabla \mathbf{v})^{\mathrm{T}}
$$

\section{Material stability analysis for continuum}

A linearized stability analysis of an infinite slab of the material follows. Such stability analysis are conventionally made since material behavior in this model problem is generally relevant to arbitrarily shaped bodies in more complex states of stress. The slab is initially at rest and in a state of uniform stress and strain. We will also call this stability result the stability of the PDE, which is equivalent to the hyperbolicity of the PDE. 
In a linearized stability analysis we investigate the stability of the slab to a perturbation in the displacement. The perturbation can be expressed as $\mathbf{x}=\overline{\mathbf{x}}+\tilde{\mathbf{x}}$ where $\tilde{\mathbf{x}}$ is a plane wave perturbation given by

$$
\tilde{\mathbf{x}}=\tilde{\mathbf{u}}=\mathbf{g e}^{\mathrm{i} \omega t+\mathrm{i} \kappa \mathbf{n}_{0} \cdot \mathbf{x}}
$$

$\omega$ is the frequency and $\mathbf{g}$ is the polarization of the wave. The perturbed deformation gradient is

$$
\mathbf{F}=\overline{\mathbf{F}}+\frac{\partial \tilde{\mathbf{u}}}{\partial \mathbf{X}}
$$

In terms of the basis $e_{i}^{*}$ the deformation gradient is

$$
\mathbf{F}^{*}=\mathbf{Q}^{\mathrm{T}} \mathbf{F} \mathbf{R}=\overline{\mathbf{F}}^{*}+\mathbf{Q}^{\mathrm{T}} \frac{\partial \tilde{\mathbf{u}}}{\partial \mathbf{X}^{*}}=\left[\begin{array}{ccc}
\lambda_{1} & 0 & 0 \\
0 & \lambda_{2} & 0 \\
0 & 0 & \lambda_{3}
\end{array}\right]+\frac{\partial \tilde{\mathbf{u}}^{*}}{\partial \mathbf{X}^{*}},
$$

where

$$
\tilde{\mathbf{u}}^{*}=\mathbf{Q}^{\mathrm{T}} \mathbf{g} \mathrm{e}^{\mathrm{i} \omega t+\mathrm{i} \kappa \mathbf{n}_{0} \cdot \mathbf{X}}=\mathbf{g}^{*} \mathrm{e}^{\mathrm{i} \omega t+\mathrm{i} \kappa \mathbf{n}_{0}^{*} \cdot \mathbf{X}^{*}} .
$$

Since $\mathbf{g}^{*}$ is an arbitrary vector in $\mathfrak{R}^{3}$ we drop the asterisk and rewrite the perturbation as $\tilde{\mathbf{u}}=\mathbf{g e}^{\mathrm{i} \omega t+\mathrm{i} \kappa \mathbf{n}_{0} \cdot \mathbf{X}}$. The corresponding deformation gradient is

$$
\mathbf{F}=\left[\begin{array}{ccc}
\lambda_{1} & 0 & 0 \\
0 & \lambda_{2} & 0 \\
0 & 0 & \lambda_{3}
\end{array}\right]+\frac{\partial \tilde{\mathbf{u}}}{\partial \mathbf{X}}
$$

The above can be used for the stability analysis for an isotropic continuum or for a particle method on an isotropic mesh with an isotropic material.

If the perturbation of the nominal stress is written as $\mathbf{P}=\overline{\mathbf{P}}+\widetilde{\mathbf{P}}$, the perturbed equations of motion (1) can be written as:

$$
\rho_{0} \ddot{\tilde{\mathbf{u}}}=\nabla_{0} \cdot \widetilde{\mathbf{P}}
$$

Here, the perturbed nominal stress is given in terms of the perturbed second PiolaKirchhoff stress and the perturbed deformation gradient as (see [5]):

$$
\begin{aligned}
\widetilde{P}_{i j} & =\widetilde{S}_{i r} F_{r j}^{\mathrm{T}}+S_{i t} \widetilde{F}_{t j}^{\mathrm{T}}=C_{i r k l}^{\mathrm{SE}} F_{a k} F_{j r} \widetilde{F}_{a l}+S_{i l} \widetilde{F}_{j l} \\
& =\left(C_{i r a l}^{\mathrm{SE}} F_{j r} F_{k a}+S_{i l} \delta_{j k}\right) \widetilde{F}_{k l}=\bar{C}_{i j k l} \widetilde{F}_{k l},
\end{aligned}
$$

where the perturbed second Piola-Kirchhoff stress tensor is given in terms of $\widetilde{\mathbf{F}}$ by

$$
\widetilde{S}_{i j}=C_{i j k l}^{\mathrm{SE}} \widetilde{E}_{k l}=C_{i j k l}^{\mathrm{SE}} F_{k a}^{\mathrm{T}} \widetilde{F}_{a l}
$$

and

$$
\bar{C}_{i j k l}=C_{i r a l}^{\mathrm{SE}} F_{j r} F_{k a}+S_{i l} \delta_{j k} .
$$


The governing equation in terms of perturbed displacements can be obtained by substituting (20) into (19):

$$
\rho_{0} \frac{\partial \tilde{u}_{i}}{\partial t^{2}}=\frac{\partial \widetilde{P}_{j i}}{\partial X_{j}}=\frac{\partial}{\partial X_{j}}\left(\bar{C}_{j i k l} \widetilde{F}_{k l}\right)=\bar{C}_{j i k l} \frac{\partial \tilde{u}_{k}}{\partial X_{j} \partial X_{l}} .
$$

For simplicity, we now consider a two-dimensional body in plane strain. Therefore, $\lambda_{3}=1$ and $\mathbf{u} \in \mathfrak{R}^{2}$. The plane wave perturbation (14) can be written in two dimensions as

$$
\tilde{\mathbf{u}}=\mathbf{g} \mathrm{e}^{\mathrm{i} \omega t+\mathrm{i} \kappa \mathbf{n}^{0} \cdot \mathbf{X}}=\left\{\begin{array}{ll}
g_{x} & g_{y}
\end{array}\right\}^{\mathrm{T}} \mathrm{e}^{\mathrm{i} \omega t+\mathrm{i} \kappa\left(X n_{1}^{0}+Y n_{2}^{0}\right)} .
$$

Substituting (24) into (23) yields

$$
\omega^{2} g_{i}-\frac{\kappa^{2}}{\rho_{0}} \bar{C}_{j i k l} n_{j}^{0} n_{l}^{0} g_{k}=0
$$

and the condition for a nontrivial solution of the above is that

$$
\operatorname{det}\left[\omega^{2} \delta_{i k}-\frac{\kappa^{2}}{\rho} \bar{C}_{j i k l} n_{j}^{0} n_{l}^{0}\right]=\operatorname{det}\left[\omega^{2} \delta_{i k}-\frac{\kappa^{2}}{\rho_{0}} A_{i k}\right]=0,
$$

where

$$
A_{i k}=\bar{C}_{j i k l} n_{j}^{0} n_{l}^{0} .
$$

The strong ellipticity condition can be expressed as

$$
\bar{C}_{j i k l} n_{j}^{0} n_{l}^{0} h_{i} h_{k}>0 \quad \forall \mathbf{h} \text { and } \mathbf{n}^{0} .
$$

If this condition is satisfied, the roots $\omega$ will be real and the continuum is stable; there are also the hyperbolicity conditions for the time-dependent PDE. Because the deformation gradient is a diagonal matrix, the right Cauchy-Green deformation tensor is

$$
\mathbf{C}=\mathbf{F}^{\mathrm{T}} \cdot \mathbf{F}=\left[\begin{array}{cc}
\lambda_{1}^{2} & 0 \\
0 & \lambda_{2}^{2}
\end{array}\right] .
$$

Substituting (29) into (7) we obtain the second Piola-Kirchhoff stress S:

$$
\mathbf{S}=\left(c_{1}+c_{2} I_{1}\right)\left[\begin{array}{ll}
1 & 0 \\
0 & 1
\end{array}\right]-c_{2}\left[\begin{array}{cc}
\lambda_{1}^{2} & 0 \\
0 & \lambda_{2}^{2}
\end{array}\right]-s_{0}\left[\begin{array}{cc}
\lambda_{1}^{-2} & 0 \\
0 & \lambda_{2}^{-2}
\end{array}\right],
$$

where $I_{1}=\lambda_{1}^{2}+\lambda_{2}^{2}+1$ and $I_{3}=\lambda_{1}^{2} \lambda_{2}^{2}$. The components of the second elasticity tensor are then

$$
\begin{aligned}
& C_{1111}^{\mathrm{SE}}=\frac{2 s_{0}-2 s_{1}}{\lambda_{1}^{4}}, \quad C_{2222}^{\mathrm{SE}}=\frac{2 s_{0}-2 s_{1}}{\lambda_{2}^{4}}, \\
& C_{1122}^{\mathrm{SE}}=C_{2211}^{\mathrm{SE}}=2 c_{2}-\frac{2 s_{1}}{\lambda_{1}^{2} \lambda_{2}^{2}}, \\
& C_{1212}^{\mathrm{SE}}=C_{2121}^{\mathrm{SE}}=C_{1221}^{\mathrm{SE}}=C_{2112}^{\mathrm{SE}}=-c_{2}+\frac{s_{0}}{\lambda_{1}^{2} \lambda_{2}^{2}} .
\end{aligned}
$$

The effective tangent modulus tensor $\overline{\mathbf{C}}$ will be given by equation (22). 


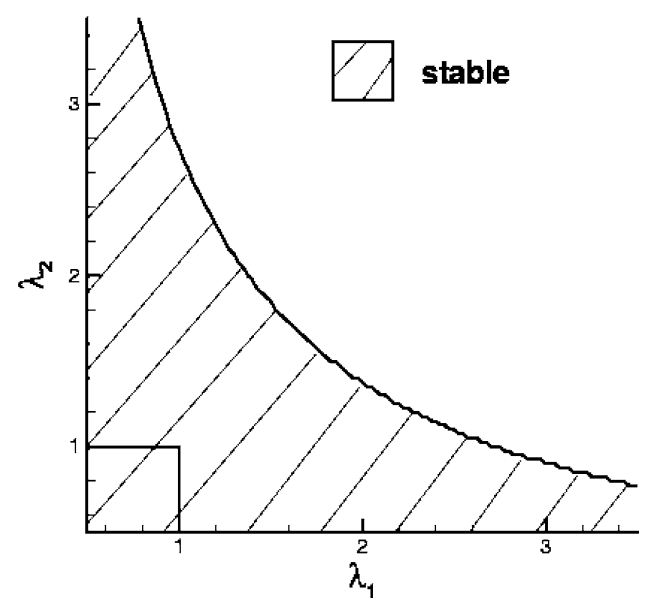

Figure 2. The stable domain of the PDE for material model in equation (5).

We consider the following material constants: $c_{1}=0.1256 \mathrm{MPa}, c_{2}=$ $0.01012 \mathrm{MPa}$ and $\lambda=10.12 \mathrm{MPa}$. In two dimensions, $n_{1}=\cos \theta$ and $n_{2}=\sin \theta$ and $\theta$ represents the wave front direction, so the stable domain of the material is given by (28) with $\mathbf{A}$ defined by

$$
\begin{aligned}
& A_{11}=\bar{C}_{1111} \cos ^{2} \theta+\bar{C}_{2112} \sin ^{2} \theta, \\
& A_{12}=\bar{C}_{1122} \cos \theta \sin \theta+\bar{C}_{2121} \cos \theta \sin \theta, \\
& A_{21}=\bar{C}_{2211} \cos \theta \sin \theta+\bar{C}_{1212} \cos \theta \sin \theta, \\
& A_{22}=\bar{C}_{1221} \cos ^{2} \theta+\bar{C}_{2222} \sin ^{2} \theta
\end{aligned}
$$

For a given deformation gradient

$$
\mathbf{F}=\left[\begin{array}{cc}
\lambda_{1} & 0 \\
0 & \lambda_{2}
\end{array}\right]
$$

if there exists $\omega_{I}$ such that $\operatorname{Im}\left(\omega_{I}\right)<0$ for any $\theta$, the material will be unstable. The stable domain for the material considered above is shown in figure 2. It can be seen that the entire compressive domain $\left(0<\lambda_{i} \leqslant 1\right)$ is stable. However, for sufficiently large extensional deformations, the material is unstable.

\section{Particle methods}

We now consider the stability of particle methods for the above hyperelastic material (5). For this purpose we first give the discrete momentum equations for a particle method. We will then examine two types of particle methods:

- Methods with a Lagrangian kernel, where the kernel (also called the smoothing function or weight function) is expressed in terms of the material (Lagrangian) coordinates, i.e. $w\left(\mathbf{X}-\mathbf{X}_{I}\right)[3,7]$. 
- Methods with an Eulerian kernel, where the kernel is expressed in term of the spatial (Eulerian) coordinates, i.e. $w\left(\mathbf{x}(t)-\mathbf{x}_{I}(t)\right)$.

Note that the nodal coordinate in the Eulerian kernel depends on time, whereas in a Lagrangian kernel it is fixed. The time dependence of this coordinate is almost always ignored in the development of the discrete particle equations, and we also ignore it.

We will consider two approaches to discretizing the equations:

- Nodal integration, where the set of quadrature points consists of only the nodes (or particles). This is equivalent to collocation with integration by parts as commonly used in SPH $[4,9,17]$.

- Stress point quadrature, in which additional quadrature points are added at the centers of the Delaunay triangulation of the particles. The idea was proposed by Dyka et al. [10,11] and it has been applied and modified by Randles et al. [24]. Note that Randles et al. only integrate on the stress points, whereas in the method we analyze the set of quadrature points includes both the particles and the stress points.

In particle methods, the dependent variables are approximated by

$$
u_{i}^{h}(\mathbf{X}, t)=\sum_{J} w_{J}(\mathbf{X}) u_{J i},
$$

where the function

$$
w_{J}(\mathbf{X})=w\left(\mathbf{X}-\mathbf{X}_{J}\right)=\frac{W\left(\mathbf{X}-\mathbf{X}_{J}\right)}{\sum_{K} W\left(\mathbf{X}-\mathbf{X}_{K}\right)}
$$

is called the kernel function. In our analysis, we use a quartic spline weight function:

$$
W(R)= \begin{cases}1-6\left(\frac{R}{R_{0}}\right)^{2}+8\left(\frac{R}{R_{0}}\right)^{3}-3\left(\frac{R}{R_{0}}\right)^{4}, & R \leqslant R_{0}, \\ 0, & R>R_{0},\end{cases}
$$

where $R=\left\|\mathbf{X}-\mathbf{X}_{J}\right\|$ and $R_{0}$ is the support radius.

We will develop the equations by a Galerkin method. Particle methods often use collocation discretizations, but the SPH methods use an integration by parts that makes it almost equivalent to a Galerkin method. For a Lagrangian kernel, the weak form of the linear momentum conservation equation is

$$
\int_{\Omega_{0}} \delta u_{i} \rho_{0} \ddot{u}_{i} \mathrm{~d} \Omega_{0}=\int_{\Omega_{0}} \delta u_{i} \rho_{0} b_{i} \mathrm{~d} \Omega_{0}-\int_{\Omega_{0}} \frac{\partial\left(\delta u_{i}\right)}{\partial X_{j}} P_{j i} \mathrm{~d} \Omega_{0}+\int_{\Gamma_{0}} \delta u_{i} \bar{t}_{i} \mathrm{~d} \Gamma_{0},
$$

where $\delta u_{i}$ is the test function and $\bar{t}_{i}$ is the boundary traction. By using the particle approximation (33), the following discrete equations are obtained

$$
m_{I} \ddot{u}_{i I}=f_{i I}^{\mathrm{ext}}-f_{i I}^{\mathrm{int}}, \quad m_{I}=\rho_{0} V_{I}^{0},
$$



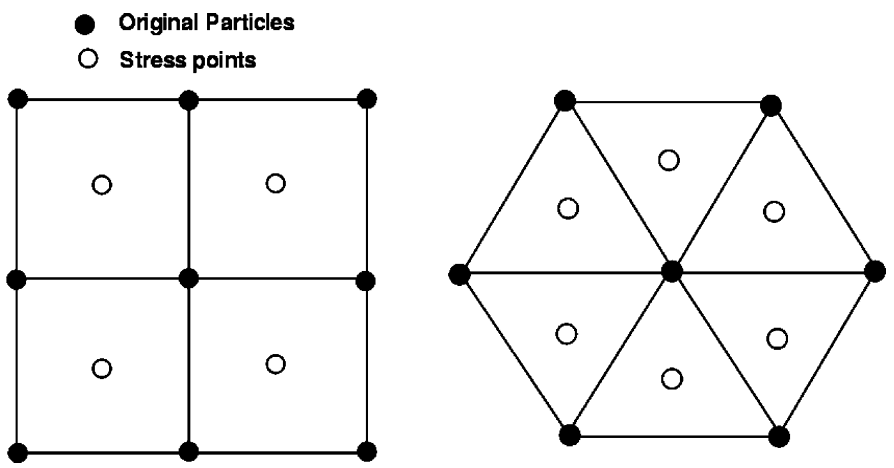

Figure 3. Square and hexagonal particle arrangements with the associated virtual elements.

where $f_{i I}^{\text {ext }}$ and $f_{i I}^{\text {int }}$ are the external and internal nodal forces, respectively, given by

$$
\begin{aligned}
f_{i I}^{\text {ext }} & =\int_{\Omega_{0}} \rho_{0} w_{I} b_{i} \mathrm{~d} \Omega_{0}+\int_{\Gamma_{0}^{t}} w_{I} \bar{t}_{i} \mathrm{~d} \Gamma_{0}, \\
f_{i I}^{\text {int }} & =\int_{\Omega_{0}} \frac{\partial w_{I}}{\partial X_{j}}(\mathbf{X}) P_{j i} \mathrm{~d} \Omega_{0} .
\end{aligned}
$$

Equation (39) can also be expressed in terms of the current domain and Cauchy stress $\sigma$

$$
f_{i I}^{\text {int }}=\int_{\Omega} w_{I, x_{j}} \sigma_{j i} \mathrm{~d} \Omega .
$$

Equation (40) is easily obtained from (39) by letting the reference domain be the current domain; see [5].

For the purpose of making an analysis of the discrete equations for the particle method feasible, a periodic arrangement of particles is selected. Figure 3 shows the two particle arrangements that are studied here. One is a square arrangement and the other is a hexagonal arrangement. When stress points are considered, they are inserted into the center of virtual squares or triangles shown in figure 3. We call these elements "virtual" since they are not explicitly needed in the discretization.

When nodal integration is used, the internal nodal forces, given by (39), are evaluated by taking the product of the integrand at the nodes with an associate volume $V_{I}^{0}$. This gives

$$
f_{i I}^{\mathrm{int}}=\sum_{J} V_{J}^{0} \frac{\partial w_{I}\left(\mathbf{X}_{J}\right)}{\partial X_{j}} P_{j i}\left(\mathbf{X}_{J}\right) .
$$

Stress points are used only for the integration (39); the resulting internal forces for a discretization with stress point are

$$
f_{i I}^{\mathrm{int}}=\sum_{J \in N_{\mathrm{M}}} V_{J}^{0 \mathrm{M}} \frac{\partial w_{I}\left(\mathbf{X}_{J}^{\mathrm{M}}\right)}{\partial X_{j}} P_{j i}\left(\mathbf{X}_{J}^{\mathrm{M}}\right)+\sum_{J \in N_{\mathrm{S}}} V_{J}^{0 \mathrm{~S}} \frac{\partial w_{I}\left(\mathbf{X}_{J}^{\mathrm{S}}\right)}{\partial X_{j}} P_{j i}\left(\mathbf{X}_{J}^{\mathrm{S}}\right),
$$


where $N_{\mathrm{M}}$ is the set of master particles and $N_{\mathrm{S}}$ is the set of slave particles which contribute to the master particle at $\mathbf{X}_{I}^{\mathrm{M}} . N_{\mathrm{S}}$ corresponds to the set of all slave particles for which $\left\|\mathbf{X}_{J}^{\mathrm{S}}-\mathbf{X}_{I}^{\mathrm{M}}\right\| \leqslant R_{0}$, i.e. the slave particles that are within the domain of influence of $\mathbf{X}_{I}^{\mathrm{M}}$, and $N_{\mathrm{M}}$ is defined similarly. The volumes $V_{J}^{0 \mathrm{M}}$ and $V_{J}^{0 \mathrm{~S}}$ are computed from a Voronoi diagram; we note that

$$
\sum_{J}^{n_{\mathrm{M}}} V_{J}^{0 \mathrm{M}}+\sum_{J}^{n_{\mathrm{S}}} V_{J}^{0 \mathrm{~S}}=V^{0} .
$$

In the above, $V^{0}$ is the total initial volume and $n_{\mathrm{M}}$ and $n_{\mathrm{S}}$ are the number of master particles and stress points in the model, respectively. Note that the volume for the master particle $I, V_{I}^{0 \mathrm{M}}$, differs from the volume associated with its mass, $V_{I}^{0}$.

\section{Stability analysis for nodal integration (standard particle method)}

\subsection{Lagrangian kernel}

The discrete perturbed momentum equations for nodal integration with a Lagrangian kernel are obtained from (36) by considering perturbations in the displacements. The perturbed momentum equation is given by

$$
m_{I} \ddot{\tilde{u}}_{i}\left(\mathbf{X}_{I}\right)=-\widetilde{f}_{i I}^{\text {int }}=-\sum_{J \in N} V_{J}^{0} w_{I, j}\left(\mathbf{X}_{J}\right) \widetilde{P}_{j i}\left(\mathbf{X}_{J}\right),
$$

where $\widetilde{P}_{i j}=\bar{C}_{i j k l} \widetilde{F}_{k l}$ and $\bar{C}_{i j k l}$ is defined by (22). Substituting (22) into (44), we obtain

$$
\omega_{i}^{2}=\frac{1}{m_{I} g_{i}} \sum_{J}\left[V_{J}^{0} w_{I, j}\left(\mathbf{X}_{J}\right) \bar{C}_{j i k l} g_{k} \sum_{K} w_{K, l}\left(\mathbf{X}_{J}\right) \cos \beta\right],
$$

where $\cos \beta=\cos \left[\kappa\left(X_{K} \cos \theta+Y_{K} \sin \theta\right)-\kappa\left(X_{I} \cos \theta+Y_{I} \sin \theta\right)\right]$. This is sometimes called a dispersion equation since it gives the wave speed for a polarization $\mathbf{g}$.

The material is stable if all frequencies are imaginary for any direction of the wave front $\theta$ under a given deformation

$$
\mathbf{F}=\left[\begin{array}{cc}
\lambda_{1} & 0 \\
0 & \lambda_{2}
\end{array}\right]
$$

Due to the rank deficiency of the discretization, an instability, usually called a spurious singular mode, occurs with nodal integration. For any given deformation, we always can find a wave number for a specific angle $\theta$ so that the frequency vanishes. This corresponds to the spurious singular mode. Even for a hexagonal arrangement of the particles, nodal integration with a Lagrangian kernel still exhibits the spurious singular mode.

Figure 4 shows the displacement in a spurious singular mode for a hexagonal arrangement of particles for $\theta=0$. The dashed line gives the hexagonal arrangement of the particles; the solid line is the displacement along the line connecting the circles (particles); the triangles identify the displacements of the particles and the line marked by these triangles is the Fourier mode. In a stable discretization, this mode would be associ- 


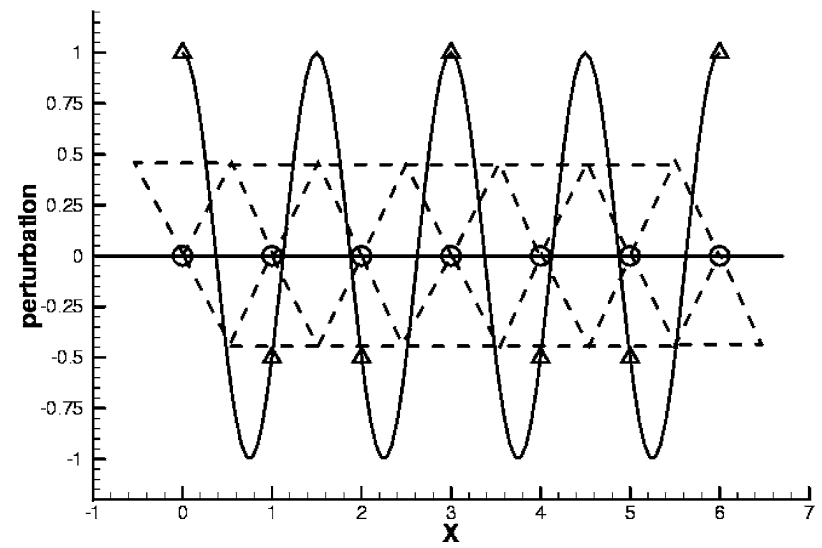

Figure 4. Spurious mode for the hexagonal arrangement on a single line of nodes; triangles identify the line that corresponds to the values of $\mathbf{u}$ at the nodes marked by circles.

ated with a high frequency, usually the cutoff frequency in the model. However, because of the rank deficiency, this mode is a zero-energy mode and its frequency vanishes.

\subsection{Eulerian kernel}

The Cauchy stress for the initial state is given by equation (13):

$$
\boldsymbol{\sigma}=\frac{1}{J} \mathbf{F} \cdot \mathbf{S} \cdot \mathbf{F}^{\mathrm{T}}=\frac{1}{\lambda_{1} \lambda_{2}}\left[\begin{array}{cc}
S_{11} \lambda_{1}^{2} & 0 \\
0 & S_{22} \lambda_{2}^{2}
\end{array}\right]
$$

and the governing equations are

$$
\rho \ddot{u}_{i}=\frac{\partial \sigma_{j i}}{\partial x_{j}} .
$$

The discrete perturbed momentum equations are

$$
m_{I} \ddot{\tilde{u}}_{i}\left(\mathbf{x}_{I}\right)=-\widetilde{f}_{i I}^{\text {int }}=-\sum_{J \in N} V_{J}^{0}\left(\widetilde{J} w_{I, j} \sigma_{j i}+J \widetilde{w}_{I, j} \sigma_{j i}+J w_{I, j} \widetilde{\sigma}_{j i}\right),
$$

where

$$
\begin{aligned}
& \widetilde{J}\left(\mathbf{x}_{J}\right)=J \delta_{s t} \sum_{K} w_{K, i}\left(\mathbf{x}_{J}\right) \tilde{u}_{s}\left(\mathbf{x}_{K}\right), \\
& \widetilde{w}_{I, j}\left(\mathbf{x}_{J}\right)=w_{I, j i}\left(\tilde{u}_{J i}-\tilde{u}_{I i}\right), \\
& \tilde{\sigma}_{i j}\left(\mathbf{x}_{J}\right)=\bar{C}_{i j k l}^{\prime} \sum_{K} w_{K, l}\left(\mathbf{x}_{J}\right) \tilde{u}_{k}\left(\mathbf{x}_{J}\right) .
\end{aligned}
$$

The effective tangent modulus tensor $\bar{C}_{i j k l}^{\prime}$ is

$$
\bar{C}_{i j k l}^{\prime}=\frac{1}{2 J}\left(C_{i j k l}^{\tau}+C_{i j l k}^{\tau}\right)-\sigma_{i j} \delta_{k l}+\sigma_{i l} \delta_{j k}+\sigma_{j l} \delta_{i k},
$$

where $C_{i j k l}^{\tau}=F_{i p} F_{j q} F_{k s} F_{l t} C_{p q s t}^{\mathrm{SE}}$. 
Letting the current configuration be the reference configuration will give $\mathbf{F}=\mathbf{I}$, $\sigma=\mathbf{S}$ and consequently that $\mathbf{C}^{\tau}=\mathbf{C}^{\mathrm{SE}}$. Substituting (49) and (50) into (48) using the perturbation of displacement given in (24), the dispersion equations for nodal integration with an Eulerian kernel are

$$
\begin{aligned}
\omega_{i}^{2}= & \frac{1}{m_{I} g_{i}} \sum_{J} V_{J}\left\{w_{I, j} \bar{C}_{j i r l}^{\prime} g_{r} \sum_{K} w_{K, l}\left(x_{J}\right) \cos \beta\right. \\
& \left.+\sigma_{j i} w_{I, j} g_{t} \sum_{K} w_{K, i}\left(x_{J}\right) \cos \beta+\sigma_{j i} w_{I, j t} g_{t}[\cos \gamma-1]\right\},
\end{aligned}
$$

where

$$
\begin{aligned}
& \cos \beta=\cos \left[\kappa\left(x_{K}-x_{I}\right) \cos \theta+\kappa\left(y_{K}-y_{I}\right) \sin \theta\right], \\
& \cos \gamma=\cos \left[\kappa\left(x_{J}-x_{I}\right) \cos \theta+\kappa\left(y_{J}-y_{I}\right) \sin \theta\right] .
\end{aligned}
$$

With an Eulerian kernel, the spurious singular mode also occurs for any given deformation. In addition, another instability is present, for $\omega$ in some cases does not vanish but has a negative imaginary part. This instability is related to the tensile instability. In any case, there is no stable domain for a particle method with nodal integration and an Eulerian kernel.

In summary, for nodal integration, both the Eulerian and Lagrangian kernels are rank deficient for the nodal patterns in figure 3 . Therefore, it is not possible to analyze the onset of material instabilities. Similar results hold for SPH.

\section{Stability analysis for stress point discretization}

\subsection{Lagrangian kernel}

For stress point quadrature with a Lagrangian kernel, the discrete perturbed momentum equations are

$$
m_{I} \ddot{\tilde{u}}_{i}\left(\mathbf{X}_{I}^{m}\right)=-\widetilde{f}_{i I}^{\mathrm{int}}=-\sum_{J \in N_{\mathrm{M}}} V_{J}^{0 \mathrm{M}} w_{I, j}\left(\mathbf{X}_{J}^{\mathrm{M}}\right) \widetilde{P}_{j i}\left(\mathbf{X}_{J}^{\mathrm{M}}\right)-\sum_{J \in N_{\mathrm{S}}} V_{J}^{0 \mathrm{~S}} w_{I, j}\left(\mathbf{X}_{J}^{\mathrm{S}}\right) \widetilde{P}_{j i}\left(\mathbf{X}_{J}^{\mathrm{S}}\right) .
$$

Using the same procedure as before the dispersion equations are:

$$
\begin{aligned}
\omega_{i}^{2}= & \frac{1}{m_{I} g_{i}} \sum_{J \in N_{\mathrm{M}}}\left[V_{J}^{0} w_{I, j}\left(\mathbf{X}_{J}^{\mathrm{M}}\right) \bar{C}_{j i k l} g_{k} \sum_{K} w_{K, l}\left(\mathbf{X}_{J}^{\mathrm{M}}\right) \cos \beta\right] \\
& +\frac{1}{m_{I} g_{i}} \sum_{J \in N_{\mathrm{S}}}\left[V_{J}^{0} w_{I, j}\left(\mathbf{X}_{J}^{\mathrm{S}}\right) \bar{C}_{j i k l} g_{k} \sum_{K} w_{K, l}\left(\mathbf{X}_{J}^{\mathrm{S}}\right) \cos \beta\right] .
\end{aligned}
$$

For the square particle arrangement we get the same instability due to rank deficiency as with nodal integration in the direction $\theta=\pi / 4$. So the use of stress points cannot eliminate the instability due to the rank deficiency for a square particle arrangement. 


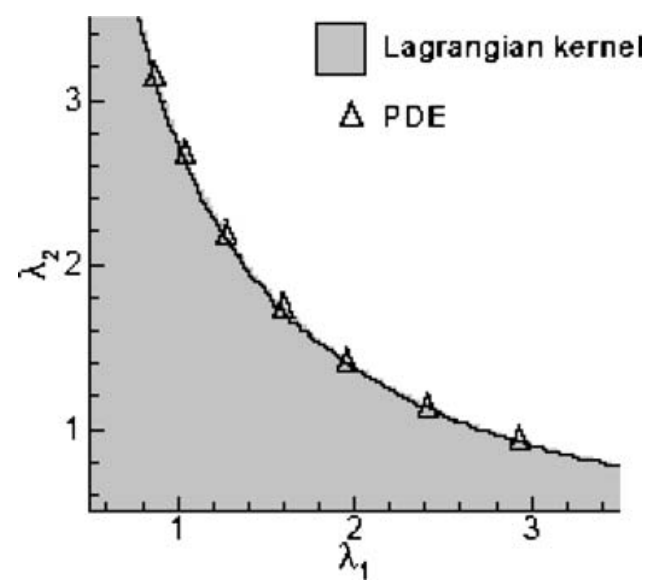

Figure 5. Stable domain for MLS particle method with stress point integration and Lagrangian kernel compared to the stable domain for the PDE.

For the hexagonal particle arrangement, stress points eliminate the spurious mode and the only instability mirrors the material instability of the continuum, as shown in figure 5. We call the stable domain as calculated for the partial differential equation the "exact" stable domain for the PDE, even though it is obtained numerically. In previous papers [7], we called it the stable domain for the continuum to distinguish it from the stable domain for the discretization. Note that the two curves, for the particle discretization and the PDE, at the scale shown are almost indistinguishable. The error between the two as measured by $\left[\sum\left(\lambda_{i}^{\text {num }}-\lambda_{i}^{\text {exact }}\right)^{2}\right]^{1 / 2}$ is a maximum of $0.1 \%$. Therefore, the particle discretization with stress points reproduces the stable domain of the material almost exactly.

Note that all states of deformation outside the shaded area in figure 5 are unstable, which means that for any state in the unshaded domain, perturbations grow unboundedly. Generally, any state beyond the boundary of the shaded domain cannot be reached in a stable process, so the boundary of the shaded domain is the line that corresponds to the onset of the instability. Nevertheless, it is interesting to observe that the material instability occurs only in part of the tensile region.

\subsection{Eulerian kernel}

For an Eulerian kernel, the dispersion equations for stress point integration are:

$$
\begin{aligned}
\omega_{i}^{2}= & \frac{1}{m_{I} g_{i}} \sum_{J \in N_{\mathrm{M}}} V_{J}^{\mathrm{M}}\left[w_{I, j} \bar{C}_{j i r l}^{\prime} g_{r} \sum_{K} w_{K, l}\left(\mathbf{x}_{J}^{\mathrm{M}}\right) \cos \beta\right. \\
& \left.+\sigma_{j i} w_{I, j} g_{t} \sum_{K} w_{K, i}\left(\mathbf{x}_{J}^{\mathrm{M}}\right) \cos \beta+\sigma_{j i} w_{I, j t} g_{t}(\cos \gamma-1)\right]
\end{aligned}
$$




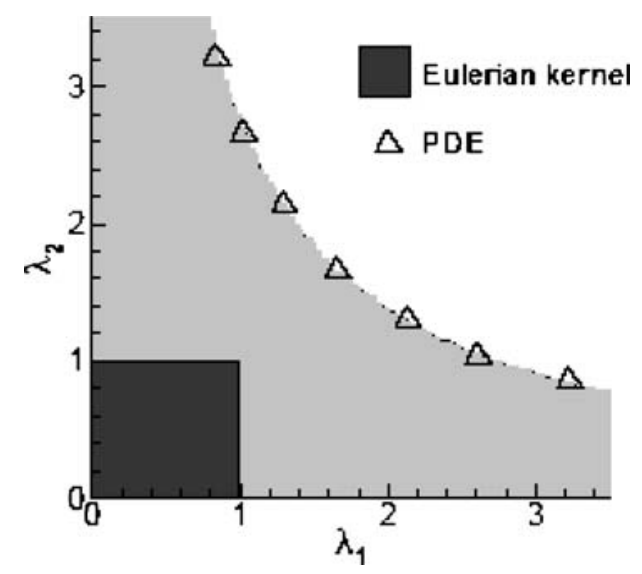

Figure 6. Stable domains of MLS particle methods for stress point integration with Eulerian kernel compared to the stable domain of the PDE.

$$
\begin{aligned}
& +\frac{1}{m_{I} g_{i}} \sum_{J \in N_{\mathrm{S}}} V_{J}^{\mathrm{S}}\left[w_{I, j} \bar{C}_{j i r l}^{\prime} g_{r} \sum_{K} w_{K, l}\left(\mathbf{x}_{J}^{\mathrm{S}}\right) \cos \beta\right. \\
& \left.+\sigma_{j i} w_{I, j} g_{t} \sum_{K} w_{K, i}\left(\mathbf{x}_{J}^{\mathrm{S}}\right) \cos \beta+\sigma_{j i} w_{I, j t} g_{t}(\cos \gamma-1)\right] .
\end{aligned}
$$

For the hexagonal arrangement, stress points eliminate the instability due to the rank deficiency for the Eulerian kernel also. Therefore, any remaining instabilities are manifestations of a discrete material instability. However, as can be see in figure 6, the domain of material stability is distorted to such a degree that we can only find a stable domain in compression. The state of compression is stable for both the continuum and the Lagrangian kernel, as shown in figure 6. Figure 7 shows the distribution of the squares of the maximum frequency $\omega$. In the domain of $\left(0<\lambda_{1}<1\right) \cap\left(0<\lambda_{2}<1\right)$ all squares of the frequencies are positive so this domain is stable. Note that by definition, $\lambda_{i}>0$ for a valid deformation.

\section{Stability analysis for Blatz-Ko material}

Now we consider a linearized stability analysis method for a Blatz-Ko material [8]. This elastic material is compressible, homogeneous and isotropic. The potential function is

$$
\psi\left(I_{1}, I_{2}, I_{3}\right)=\frac{\mu}{2}\left(I_{2} J^{-2}+2 J-5\right),
$$

where $J=\sqrt{I_{3}}$ and $\mu$ is a constant; we will use $\mu=32$ psi. The second Piola-Kirchhoff stress and the second elasticity tensor can be obtained from the potential energy by (56):

$$
\mathbf{S}=2 \frac{\partial \psi}{\partial \mathbf{C}}=\mu\left[I_{3}^{-1} I_{1} \mathbf{I}-I_{3}^{-1} \mathbf{C}-\left(I_{2} I_{3}^{-1}-I_{3}^{1 / 2}\right) \mathbf{C}^{-1}\right],
$$




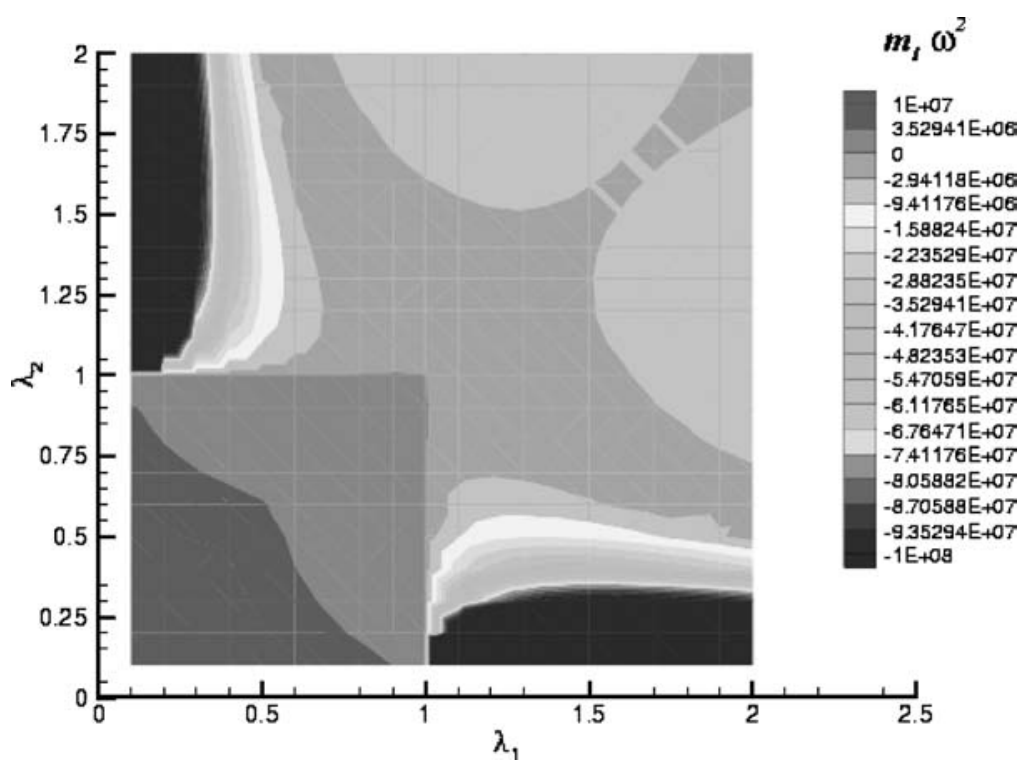

Figure 7. Distribution of frequency $\omega^{2}$ for particle method with stress points and Eulerian kernel.

$$
\begin{aligned}
\mathbf{C}^{\mathrm{SE}}=2 \frac{\partial \mathbf{S}}{\partial \mathbf{C}=} & 2 \mu\left[I_{3}^{-1} \mathbf{I} \otimes \mathbf{I}-I_{3}^{-1} I_{1} \mathbf{C}^{-1} \otimes \mathbf{I}-I_{3}^{-1} \mathbf{I}+I_{3}^{-1} \mathbf{C}^{-1} \otimes \mathbf{C}\right] \\
& -\left(I_{3}^{-1} I_{1} \mathbf{I}-I_{3}^{-1} \mathbf{C}-I_{2} I_{3}^{-1} \mathbf{C}^{-1}-\frac{1}{2} I_{3}^{1 / 2} \mathbf{C}^{-1}\right) \otimes \mathbf{C}^{-1} \\
& -\left(I_{2} I_{3}^{-1}-I_{2}^{1 / 2}\right) \frac{\partial \mathbf{C}^{-1}}{\partial \mathbf{C}} .
\end{aligned}
$$

Knowles and Sternberg [16] obtained the stable domain for the Blatz-Ko material by an analysis of the associated issue of ellipticity (i.e. loss of ellipticity which corresponds to material instability in static (equilibrium) processes). The displacement equations of equilibrium were found to be locally elliptic when the following inequalities are satisfied:

$$
\gamma<\frac{\lambda_{i}}{\lambda_{j}}<\frac{1}{\gamma}, \quad i \neq j, \quad \gamma=2-\sqrt{3} \cong 0.268 .
$$

The stable domain obtained by taking Knowles and Sternberg's results and setting $\lambda_{3}=1$ is shown in figure 8 and corresponds to

$$
\gamma<\lambda_{1}<\frac{1}{\gamma}, \quad \gamma<\lambda_{2}<\frac{1}{\gamma}, \quad \gamma<\frac{\lambda_{1}}{\lambda_{2}}<\frac{1}{\gamma} .
$$

However, they are based on a three-dimensional perturbation

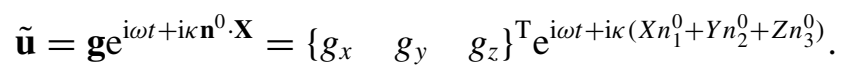




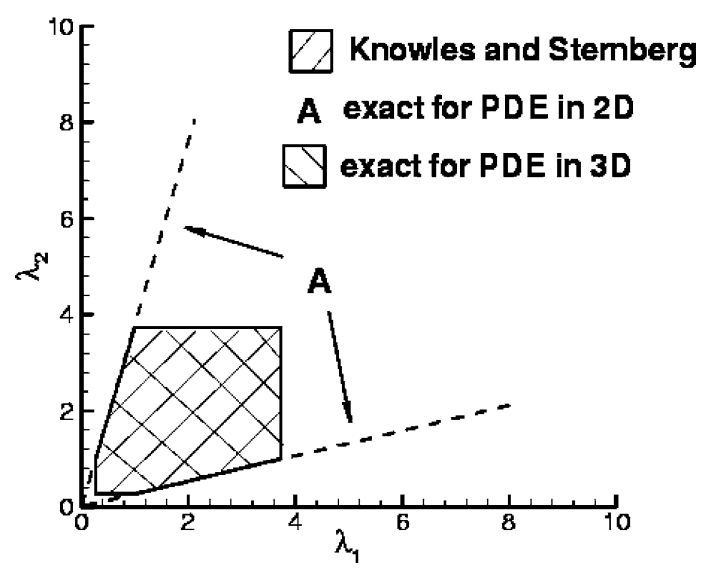

Figure 8. Stable domain of Blatz-Ko material.
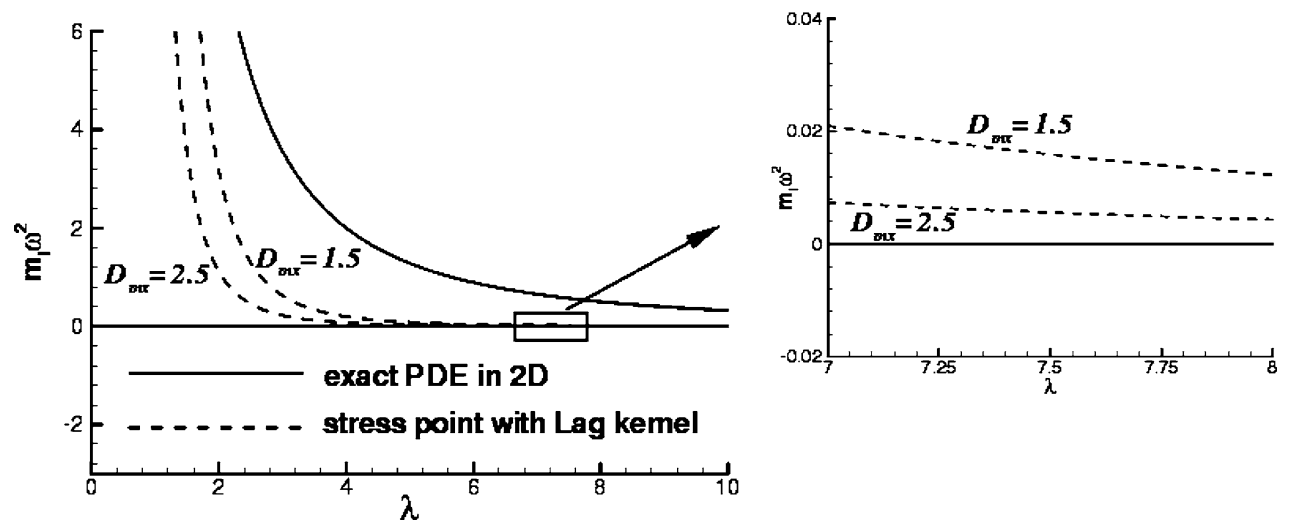

Figure 9. Frequency $\omega^{2}$ for $\lambda_{1}=\lambda_{2}=\lambda$.

In our analysis, the perturbations are two-dimensional, which leads to a different domain of stability. The result is shown in figure 8 and corresponds to

$$
\gamma<\frac{\lambda_{1}}{\lambda_{2}}<\frac{1}{\gamma}
$$

In examining the stability of particle methods with Lagrangian and Eulerian kernels for the Blatz-Ko material, only the hexagonal arrangement of particles with stress points is considered here since we expect the square arrangement to be rank deficient; rank deficiency is independent of the material model. These studies are restricted to $2 \mathrm{D}$ as in the previous example. The stable domain from the particle method with the Lagrangian kernel is identical to the 2D stable domain of the PDE. However, we found that the frequencies are close to zero for states of large deformation, i.e. large $\lambda_{i}$. Figure 9 shows that the system is close to unstable when the deformations are large even when 


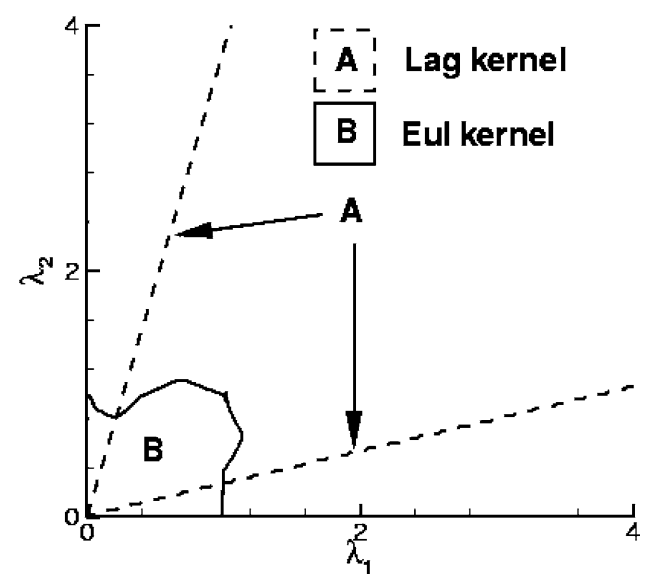

Figure 10. Stable domains for MLS particle methods with stress points for Eulerian and Lagrangian kernels.

a Lagrangian kernel is used. Figure 9 shows that the frequencies are closer to zero for larger support size, $D_{m x}=2.5$. This was also mentioned in [7].

Figure 10 compares the stable domains of the particle method for Eulerian and Lagrangian kernels. It clearly shows that the Eulerian kernel severely distorts the domain of material stability, particularly for tensile deformations.

\section{Conclusion}

Material instability is an essential feature of a material model, for it enables the simulation of phenomena such as fracture and shear bands. As shown in [1], material instabilities in rate independent materials result in a localization of the deformation to a set of measure zero; similar behavior has been observed in multi-dimensions. In dynamics, it corresponds to the loss of hyperbolicity.

Particle methods with nodal integration have two sources of instabilities: (1) numerical instability due to rank deficiency of the discretization; (2) instabilities that correspond to the material instability.

For nodal integration, both Eulerian and Lagrangian kernels are unstable. This instability is primarily due to the rank deficiency of the discrete equations. Since particle methods have been widely used, and they are all essentially of the "nodal" integration form, this result is quite surprising. Most likely, these instabilities are ameliorated by the viscosities that are commonly used. Very few rigorous studies of the accuracy have been made, and high frequency error in numerical solutions has probably gone undetected. Furthermore, it is possible that the instability in SPH is a saddle-point in a valley, so that the growing oscillations transition the solution to a stable neighborhood.

For stress point schemes, particle methods in two dimensions are not rank deficient for triangular node arrangements. It is then possible to study how well the domain of material instability is reproduced by the particle methods. Unfortunately, the generality of such studies is limited because a stability analysis pertains a specific material. We 
considered a hyperelastic material similar to the Mooney-Rivlin material and a Blatz-Ko material. In both materials, the Eulerian kernel severely distort the domain of material stability. This implies standard SPH methods would be very erroneous in predictions of shear bands, void formation and tearing (cracking). On the other hand, the Lagrangian kernel reproduces the domain of material stability with exceptional fidelity. This implies that it would be much more suitable for modeling material failure phenomena.

Unfortunately, the nature of Lagrangian kernels impairs an intrinsic advantage of particles methods: the ability to model very large distortions. Lagrangian kernels are not able to treat flows of fluids and would likely fail for very large deformations of solids [22]. However, we have found that Lagrangian kernels are quite effective for the deformations found in many solid mechanics problems involving failure.

The results obtained by Randles et al. [24] differ from ours in that they conclude that a particle method is stable when it is linear complete. The difference in conclusions may arise from their assumption of a linear isotropic material response; we have considered a material that possesses an unstable domain. It is worth noting that most (if not all) objective material models will posses domains of instability. It is also puzzling that their analysis did not over reveal the instability due to rank deficiency. This requires further study.

\section{Acknowledgements}

The support of the Office of Naval Research and the Army Research Office are gratefully acknowledged.

\section{References}

[1] Z. Bazant, T. Belytschko and T.P. Cheng, Continuum theory for strain-softening, J. Engrg. Mech. 110 (1984) 1666-1692.

[2] S. Beissel and T. Belytschko, Nodal integration of the element-free Galerkin method, Comput. Methods Appl. Mech. Engrg. 139 (1996) 49-74.

[3] T. Belytschko, Y. Guo, W.K. Liu and S.P. Xiao, A unified stability analysis of meshless particle methods, Internat. J. Numer. Methods Engrg. 48 (2000) 1359.

[4] T. Belytschko, K. Krongauz, D. Organ, M. Fleming and P. Krysl, Meshless methods: An overview and recent developments, Comput. Methods Appl. Mech. Engrg. 139 (1996) 3-47.

[5] T. Belytschko, W.K. Liu and B. Moran, Nonlinear Finite Elements for Continua and Structures (Wiley, New York, 2000).

[6] T. Belytschko, Y.Y. Lu and L. Gu, Element-free Galerkin methods, Internat. J. Numer. Methods Engrg. 37 (1994) 229-256.

[7] T. Belytschko and S.P. Xiao, Stability analysis of particle methods with corrected derivatives, Comput. Math. Appl. 43 (2002) 329-350.

[8] P.J. Blatz and W.L. Ko, Application of finite elastic theory to the deformation of rubbery materials, Trans. Soc. Rheol. 6 (1962) 223.

[9] J. Dolbow and T. Belytschko, Numerical integration of the Galerkin weak form in meshfree methods, Comput. Mech. 23 (1999) 219-230.

[10] C.T. Dyka and R.P. Ingel, An approach for tension instability in smoothed particle hydrodynamics (SPH), Comput. Struct. 57 (1995) 573-580. 
[11] C.T. Dyka, P.W. Randles and R.P. Ingel, Stress points for tension instability in SPH, Internat. J. Numer. Methods Engrg. 40 (1997) 2325-2341.

[12] D.P. Flanagan and T. Belytschko, A uniform strain hexahedron and quadrilateral with orthogonal hourglass control, Internat. J. Numer. Methods Engrg. 17 (1981) 679-706.

[13] J. Hadamard, Leçons sur la Propagation des Ondes et les Equations de l'Hydrodynamique (Hermann, Paris, 1903) chapter 6.

[14] R. Hill, Acceleration waves in solids, J. Mech. Phys. Solids 10 (1962) 1-16.

[15] J.F. Kalthoff, Modes of dynamic shear failure in solid, Internat. J. Fract. 101 (2000) 1-31.

[16] J.K. Knowles and E. Sternberg, On the ellipticity of the equations of nonlinear elastostatics for a special material, J. Elasticity 5(3/4) (1975) 341-361.

[17] K. Krongauz and T. Belytschko, Consistent pseudo-derivatives in meshless methods, Comput. Methods Appl. Mech. Engrg. 146 (1998) 371-386.

[18] W.K. Liu, S.F. Li and T. Belytschko, Moving least square reproducing kernel method. (I) Methodology and convergence, Comput. Methods Appl. Mech. Engrg. 143 (1997) 113-154.

[19] A. Marchand and J. Duffy, An experimental study of the formation process of adiabatic shear bands in a structural steel, J. Mech. Phys. Solids 38 (1988) 251-283.

[20] J.J. Monaghan, An introduction to SPH, Comput. Phys. Comm. 48 (1988) 89-96.

[21] R.W. Ogden, Nonlinear Elastic Deformations (Ellis Horwood, Chichester).

[22] T. Rabczuk, T. Belytschko and S.P. Xiao, Stable particle methods based on Lagrangian kernels, J. Numer. Methods Engrg., accepted.

[23] P. Randles and L. Libersky, Smoothed particle hydrodynamics: Some recent improvements and applications, Comput. Methods Appl. Mech. Engrg. 146 (1968) 371-386.

[24] P.W. Randles, A.G. Petschek, L.D. Libersky and C.T. Dyka, Stability of DPD and SPH, in: Lecture Notes in Computer Science, Vol. 26 (Springer, New York, 2002) pp. 339-358.

[25] J.R. Rice, Inelastic constitutive relations for solids: Internal-variable theory and its application to metal plasticity, J. Mech. Phys. Solids 19 (1971) 443-455.

[26] J.W. Rudnicki and J.R. Rice, Conditions for localization in pressure sensitive dilatant materials, J. Mech. Phys. Solids 23 (1975) 371-394.

[27] J.W. Swegle, D.L. Hicks and S.W. Attaway, Smoothed particle hydrodynamics stability analysis, J. Comput. Phys. 116 (1995) 123-134. 